\title{
Assessment of the Management Tools of Electric Systems in Developing Countries
}

This paper was downloaded from TechRxiv (https://www.techrxiv.org).

LICENSE

CC BY 4.0

SUBMISSION DATE / POSTED DATE

$16-11-2021 / 29-11-2021$

CITATION

Diop, Moustapha; Kebe, Baye (2021): Assessment of the Management Tools of Electric Systems in Developing Countries. TechRxiv. Preprint. https://doi.org/10.36227/techrxiv.17024816.v1

$\mathrm{DOI}$

10.36227/techrxiv.17024816.v1 


\title{
Assessment of the Management Tools of Electric Systems in Developing Countries
}

\author{
Baye Kebe, Moustapha Diop, Member, IEEE
}

\begin{abstract}
This article assesses the management tools of electric systems in developing countries. To promote the nuclear energy as an alternative resource in developing countries, the International Atomic Energy Agency (I.A.E.A.) has encouraged the development of a decision-making tool which optimizes operational activities in electric systems. Since 1982, most developing countries use that tool or a derivative one; recurrent management difficulties are encountered despite the numerous studies and the efforts to enhance the management performances. Activities are the main levers in the management of electric systems. They are set to improve the performances which translate the efficiencies. In dealing with the difficulties, none of the previous studies has considered the tool assessment. Monte Carlo or probabilistic or derating simulation methods are used in the tool Implementation; simulations are only relevant if models are realistic and the systems properly analysed. We show in this paper indirectly from the WASP notebook guide A (I.A.E.A.) 1984 and directly from the evolution of electrical system's characteristics that the electric systems models are not suitable in developing countries. An unrealistic model stands for a system significantly different from the actual one; the differences lead to reference quantity value management difficulties and to the impossibility to set up the infinitesimal evolution responsible for the overall behaviour of the system and underlies the implementation of the tool. The article puts the constraints and associated activities at the centre of electrical system management, it opens prospects for efficiency management with a significant impact on upcoming electric systems.
\end{abstract}

Index Terms-Assessment, Tool, Irrelevant, Enhancement, Performance

\section{INTRODUCTION}

$\mathbf{T}$ HE electric systems are dynamic systems, their configurations and states are modulated by the levels of the electricity demand and the implemented activities. Their management objectives are to meet the electricity demand, in an area, with a satisfactory level of reliability at the lowest cost. The costs of inputs and other resources are very sensitive with the activity levels and the procurement contexts; the system is managed with great uncertainties, the management must be planned and flexible. To achieve stated performances, all the activities must, somehow, be motivated; it is then necessary to have decision-making tools which allow to quantitatively define the characteristics of the activities that match the stated performances.

Before the oil crisis of 1973 , there was no decision-making tool in developing countries, the electric systems management was qualitative and empirical; no major management difficulties were experienced.

The oil crisis has led to a sharp increase in operating and electricity costs, the electricity prices did not follow the pace; leading consequently to cash flow difficulties in utility companies. The then state-owned companies were unable to

This paragraph of the first footnote will contain the date on which you submitted your paper for review. It will also contain support information, including sponsor and financial support acknowledgment. For example, "This work was supported in part by the U.S. Department of Commerce under Grant BS123456",

The next few paragraphs should contain the authors' current affiliations, including current address and e-mail. For example, F. A. Author is with the National Institute of Standards and Technology, Boulder, CO 80305 USA (e-mail: author@boulder.nist.gov).

B. Kebe is retired (2010) from the generation Department the senegalese utility compagny (Seneelec - 28 rue vincent Dakar Senegal) and a former professor at the interafrican Electrical Engineering College (IEEC) in Bingerville in Cote d'Ivoire - R.C.I.. He is currently an Energy Consultant (e-mail:bayeyame2@gmail.com).

M. Diop is with the STI Department, Cheikh Anta Diop University of Senegal, BP 5004 Dakar-Fann, Senegal (e-mail: moustapha17.diop@ucad.edu.sn). meet the operational requirements and ensure the investments needed to secure the system's expansion with more expensive alternative energy resources.

To promote civil nuclear power, as one of the alternatives in the electric system expansion in developing countries, the International Agency Atomic Energy (I.A.A.E.), relying on Tennessee Valley Authority (TVA) a utility company and Argonne National Laboratory (A.R.N.L.) a research laboratory both in the United States, has supported the development of a decision-making tool for electrical systems management. The tool referred as "Wien Automatic System Planning (WASP)" [1],[2] simulates operational activities in the electric system and takes into account all forms of primary energy resources including civil nuclear energy. Two main management indexes, the reliability of the supply-demand system and the cost, are considered and calculated.

The exchanges between the Agency and state members has led to several improvements of the original WASP codes. Between 1975 and 1981, engineers and energy system planners from 43 developing countries and international organizations have been trained by the Agency in the use of the WASP planning methods. In 1982, the WASP programs were transferred to 45 applicant countries which started using them to manage their respective electric systems. Until now the planning, the management and the regulatory decisions in the electricity sectors of developing countries are based on either the "WASP" or on a derivative tools.

The first management exercises using the WASP tool, from 1982 to 1990 , were already marked with recurrent difficulties; instead of associating those difficulties with the newly developed tool which then should be improved; they were associated solely to a lack of motivation and leadership of the leaders in the system unable to implement the reforms needed to liberalize the sector and ensure its dynamism.

The sector has been liberalized since, and most of the nec- 
essary institutional and structural reforms have been implemented. The recurrent difficulties still persist despite the numerous studies, audits, and other action plans implemented to enhance management performances. They are evidenced by the high electricity costs, the low levels of system reliability of electric systems and the important and needed supports from the state development partners and the state subsidies.

Operational activities are the main management levers in electric system, but none of the previous audits or diagnostics took into account the assessment of the management tool. This article completes therefore the previous studies.

\section{THE METHOD OF THE TOOL AND ITS RELEVANCE}

Simulations are one of the most effective methods used to study dynamic systems; they consists, with the knowledge of the system, to make a model which is its abstract and simpler representation and stands for it. The model can be artificially and gradually evolved; the system is studied by simulations through the model, to understand and predict its behaviour or drive it to a previously defined state.

Monte Carlo [3], Probabilistic [2], [4] or derating simulation methods are used to calculate the management indexes. Simulations are only relevant if these three conditions are met:

- The system is analysed properly in order to bring out the accurate interactions with its environment and the infinitesimal evolution leading to the overall behaviour of the system.

- The model is realistic, which means it is a satisfactory approximation of the actual system [5].

- The management indexes are characteristics of the systems, then related to its state quantities.

. If these conditions are met, the tool developed and supported by the Agency (I.A.A.E.) is relevant in its principle and its methods; the assessment of the tool must go beyond the principle and the methods and reach its implementation. We have re-examined the tool implementation to see if the conditions stated above are actually met.

The assessment is aimed to link the recurrent management difficulties in developing countries (P.V.D.) with the tool's implementation.

\section{ASSESSMENT OF THE TOOL IMPLEMENTATION}

\section{A. Generalities}

\section{1) The system boundary and infinitesimal evolution}

A system is under the influence of its environment (boundary), which therefore has to be defined as accurately as possible to lead to its real evolution or behaviour.

The electricity is provided to $n_{g}$ independent groups of consumers through cables and wires with different voltage levels, the demand value and the energy loss rates $\eta_{g}$ are different from one voltage level $\mathrm{g}$ to another. The system boundary has $n_{g}$ independent components, for simulation purposes, it is necessary to consider separately the corresponding $D_{g}(\mathrm{t})$ demand values.

At an instant $\mathrm{t}$ of a period of interest $\mathrm{T}$, if $\mathrm{g}$ is a demand component index, the global demand $D_{g l b}(\mathrm{t})$ is:

In the consumer side

$$
D_{g l b}(t)=\sum_{g=1}^{g=n_{g}} D_{g}(t)
$$

In the generation side

$$
D_{g l b}(t)=\sum_{g=1}^{g=n_{g}} \frac{D_{g}(t)}{\eta_{g}}
$$

$D_{g l b}(\mathrm{t})$ is on each side the sum of $n_{g}$ random and independent terms respectively equal to $D_{g}(t)$ and $\frac{D_{g}(t)}{\eta_{g}}$.

\section{2) The infinitesimal evolution of the system}

Let $\mathrm{t}, \mathrm{S}(\mathrm{t})$ and $V_{k}(t)$ be respectively a time instant, a dynamic system in equilibrium at $\mathrm{t}$ and its different system state quantity values $(\mathrm{k}=1,2,3, \ldots)$; if $\mathrm{u}(\mathrm{t})$ is the resulting interaction between $\mathrm{S}(\mathrm{t})$ and its environment and if $E_{0}(\mathrm{t})$ is the interaction free reference state, the knowledge of $E_{0}(\mathrm{t})$ and $\mathrm{u}(\mathrm{t})$ unambiguously determines the state of $\mathrm{S}(\mathrm{t})$ at the time instant $\mathrm{t}$.

$$
\begin{aligned}
& \text { Resulting interaction system } \mathbf{S}(\mathrm{t}) \text { - Environment : } \mathrm{U}(\mathrm{t}) \\
& \text { Isolated system } U(t)=0 \quad \text { Non isolated system } U(t) \neq 0 \\
& S(t)=\left(E_{0}(t), V k_{0}(t)\right) \quad U(t) \Rightarrow S(t)=(E(t), V k(t)) \\
& V_{k}(t)=V_{k r f}(t)+I m_{v k} u(t)
\end{aligned}
$$

$V_{k r f}(\mathrm{t})$ and $I m_{v k} \mathrm{u}(\mathrm{t})$ are respectively the value of $V_{k}(\mathrm{t})$ when the state of $\mathrm{S}(\mathrm{t})$ is $E_{0}(\mathrm{t})$ and the impact of $\mathrm{u}(\mathrm{t})$ on the state quantity $V_{k}(\mathrm{t})$ The equation above means; if the reference state of a system $E_{0}(\mathrm{t})$ is known in a period of interest $\mathrm{T}$, the evolution of $\mathrm{S}(\mathrm{t})$ in that period is completely determined by the knowledge of the succession of the resulting interactions $u(t)$.

\section{3) The model of the system}

If $\mathrm{T}=[\mathrm{t} 1, \mathrm{t} 2]$ is a time of interest period and $\mathrm{u}(\mathrm{t})$ is the resulting interaction at any time $\mathrm{t} \in \mathrm{T}$, a state quantity values $V_{k}(t)$ consolidates in $\mathrm{T}$ the management value $G Q_{k}(\mathrm{~T})$ which is equal to the sum of all $V_{k}(t)$ values in T.

$$
G Q_{k}(T)=\int_{t 1}^{t 2} V_{k r f}(t) d t+\int_{t 1}^{t 2} \operatorname{Im}_{v k} u(t) d t
$$

The behaviour of the system is an input in the management tool; because it is assumed that the state quantity value at any time in the period of interest is constant and equal to their average value.

$$
V_{k}(t)=\frac{\int_{t 1}^{t 2} V_{k r f}(t) d t+\int_{t 1}^{t 2} I m_{v k} u(t) d t}{T}
$$




\section{4) The management indexes}

The two main management quantities are the reliability $\epsilon(\mathrm{T})$ and the cost $\cos (\mathrm{T})$.

The reliability is related to the instantaneous unserved energy END (t) which is calculated as:

$$
\operatorname{END}(t)=\operatorname{Max}\left\{0 ;\left(V_{1}(t)-V_{2}(t)\right)\right.
$$

$V_{1}(\mathrm{t})$ and $\left.V_{2}(\mathrm{t})\right)$ are respectively the instantaneous unavailable capacity and demand level. The END (t) values consolidate in the time period $\mathrm{T}$, the unserved energy $\operatorname{END}(\mathrm{T})$ and the reliability off the supply-demand system $\epsilon(\mathrm{T})$ is:

$$
\epsilon(T)=\frac{\operatorname{END}(T)}{\operatorname{Demand}(T)}
$$

Demand $(\mathrm{T})$ is the management value consolidated by Demand (t) in the time period $\mathrm{T}$.

The instantaneous cost value cost (t) is a linear combination of the quantities consumed per time unit which are state quantities; it consolidates in $\mathrm{T}$ the management quantity value $\cos (\mathrm{T})$ The two main management indexes are both directly related to state quantities; they evolve like the state of the electric system with operational activities. They can be calculated and monitored using equations like equation 4

The assessment will be supported by two independent approaches, the first is indirect and based on the tool notebook guide [6]; the second is direct and based on the evolution of the characteristics of the electric system with time.

\section{B. the Assessment}

\section{1) Indirect approach}

The WASP note book guide $\mathrm{A}[\overline{6}]$ is a detailed presentation of the management tool from the first stage of its design to the feedback following its implementation. Three important aspects of the WASP tool presentation are considered:

- the specifications of the system and its model;

- The management indexes and their calculations;

- the feedback following the implementation of the tool. to see if what is basically expected from the generality have been properly implemented in the tool.

a) The system its specifications and its model: The system specification

- The system specification The generation units are specified to meet an electric demand which must be in terms of power, energy or load variation for a time of the interest period $\mathrm{T}$ within a year, such as an hour, a month or several months.

The $D_{g}(\mathrm{t})$ demand values are, with operational activities, parts of the interactions of the system with its environment. The $n_{g}$ instantaneous energy demands values $D_{g}(t)$ and $\frac{D_{g}(t)}{\eta_{g}}$ respectively on the consumer and the generation sides. They consolidate in the time period $\mathrm{T}$ corresponding management values $D_{g}(T)$ and $\frac{D_{g}(T)}{\eta_{g}}$.

The global demand management values are the sum of the $n_{g}$ management values and are:

On the generation side:

$$
D_{g l b}(T)=\sum_{g=1}^{g=n_{g}} \frac{D_{g}(T)}{\eta_{g}}
$$

On the consumer side:

$$
D_{g l b}(T)=\sum_{g=1}^{g=n_{g}} D_{g}(T)
$$

The Model of Analysis of the Energy Demand (M.A.E.D.)[8] converts the yearly value $(\mathrm{T}=8760 \mathrm{~h})$ of the global demand management value $D_{g l b}(T)$ into hourly demand values to feed the management tool for optimization purposes. In doing so, it is implicitly assumed that the $n_{g} D_{g}(t)$ demand values of the $n_{g}$ groups of consumers are not random and independent. This assumption is not true since all individual demands are random and independent; it has an impact on the simulations which is related to the relative importance of $D_{g}(\mathrm{~T})$ components and the respective variations of $D_{g}(t)$ values in the time of interest period $\mathrm{T}$.

- The system model

Any period of interest $\mathrm{T}=[\mathrm{t} 1, \mathrm{t} 2]$ is composed of succession nq Ti intervals such as; if ui(t) $=0$ in the interval $\mathrm{i}, u i(t) \neq 0$ in the next interval $i+1$ and vice versa.

A more precise calculation of the mean value of the state quantity $\overline{V_{k}(t)}$ in $\mathrm{T}$ is then:

$$
\overline{V_{k}(t)}=\frac{\sum_{t 1}^{t 2} V_{k r f}(t) d t+\sum_{i=1}^{i=n q} \sum_{t i}^{t i+1)} \operatorname{Im}_{v k} u(t) d t}{T}
$$

Based on an approximation, the model assigns to the time dependant state variables values $V_{k}(\mathrm{t})$ a constant values equal to their average values $\overline{V_{k}(t)}$ in the period of interest $\mathrm{T}$ :

$$
V_{k}(t)=\overline{V_{k}(t)}
$$

This assignment for a dynamic system has objective grounds or is true, only under certain conditions called within the "validity limits" of the approximation which is for all $\mathrm{t}$ in $\mathrm{T}$ :

$$
\frac{V_{k}(t)-\overline{V_{k}(t)}}{V_{k}(t)} \approx 0
$$

. Those conditions have to be specified for the model to be realistic and the simulations to be relevant.

The "validity limits" are not explicitly specified in the whole presentation of the notebook guide. The model and the simulations have to be respectively considered unrealistic and irrelevant.

Another consequence with the assignment is, the state quantity values are constant in the time of interest which means the system is invariant and there are no infinitesimal evolution.

b) The tool management indexes and their calculations: The overall goal of the management of an electric system is to provide a service of electricity with an acceptable level of reliability at a minimum cost.

The most widely used reliability index is called "Loss Of Load Probability (LOLP)" often used to calculate also the generation costs. The "LOLP" is deduced successively from the chronological load curve and the rearrangement of its points to obtain the Load Duration Curve (LDC); the LDC is then normalised to obtain the "LOLP" curve. The "LOLP" is a very misunderstood index because: 
- The "LOLP" It is not a probability; it is not also a system characteristic, since its value is a function of the segmentation of the period of interest. An attempt to clarify this confusion] has led to the definition of the Loss of Load Expectation (LOLE) curve by BILLINTON [7]

- The "LOLP" is independent from the generation system and solely related to the load evolution; it cannot therefore account for loss of loads in the electric system.

The "LOLP", unlike the actual reliability index, is not an electric system characteristic or is not related to a state function. It cannot be used to calculate the actual reliability of the electric system.

c) The feedback following the implementation the WASP [6]: The limits of the WASP programs has been implicitly declined in the note book guide of the tool, as a feed-back following its implementation. They were put down as follows for electric systems with a large component of thermal units: " The WASP code may also produce an inaccurate representation of the system operations for power systems relatively small and composed of a small number of thermal units only

Two different system entities are referred to in the statement of the limits, the generating system as a whole and the individual power generator units.

Two relations are supposed to set the limits:

- The system is modest (i.e. the system is small)

- The number of individual units is small

These two relations are both undefined, the above declined limits do not have a practical or physical meaning; the statement should then be corrected.

A limit of a system is one of its characteristic; consequently, like all state quantities of a system, they must be stated as functions of the state variables.

Let n, $C_{A}$ and $C a_{i}$, be respectively, the number of individual units of the system, the electric power values of the whole system and that of the elementary unit $\mathrm{i}(n \geq 1)$.

If $C a_{M}=\max \left\{C a_{i}\right\}$, the ratio $\frac{C a_{M}}{C A}$ is the order of magnitude of the impacts of operational activities on the state quantities of the system.

Since the limit expression is related to the impact of operational activities, the reformulation of the corresponding limit which have a physical and a practical meaning should be stated as follows:

"The WASP program system may be inappropriate to account for operational activities in production systems when the activities have a significant impact on its state quantities"

Which also means, for the capacity, when $\frac{C a_{M}}{C A}$ is a significant number

In developing countries, the electric power of the larger single units are significant compared to the system's electric power; then, $\frac{C a_{M}}{C A}$ is significant compared to the corresponding $\frac{C a_{M}}{C A}$ values of highly interconnected electric systems in developed countries.

The limits of the WASP declined as a feedback from its implementation, implicitly feature the electric systems in developing countries outside the scope of use of the WASP programs.

\section{2) The Direct Approach}

The management of an electrical system depends on its reliability level which is a result of the compliance with good management practices and on the management tool's quality. The reliability of the generating system is measured by the rate of the installed capacity available; it can be spotted on the axe on fig 1 oriented from worst to better.

worst better

Fig. 1. Reliability evolution axis

a) The two reliability levels of an electric system: Depending on the quality of the investments and the compliance with operating requirement, the system has two characteristic reliability levels:

- A reference level $S_{\text {ref }}$ Within good management practices, the electric power of a system, its different units and their characteristics are chosen considering the electricity demand level values and their evolution with time. When more over the operating requirements given by the manufacturers are met, the system stays reliable with time; the impacts of the constraints on the state quantity values are negligible. Considering the electric power, that means if $\delta c_{i}(t)$ is the unavailable capacity of unit $i$ at a given instant $t$, that the unavailable and available capacities of the system are respectively:

$$
\begin{gathered}
C_{\text {inds }}(t)=\sum_{i}^{n} \delta c_{i}(t) \\
C A_{\text {disp }}(t)=\sum_{i}^{n} c_{i}(t)=C_{A}-\sum_{i}^{n} \delta c_{i}(t)
\end{gathered}
$$

The impact of constraints on the available capacity are insignificant means:

$$
I_{m p}(t)=\frac{\sum_{i}^{n} \delta c_{i}}{C_{A}}<<1
$$

The system is then said to be on its reference level $\left(S_{r e f}\right)$,

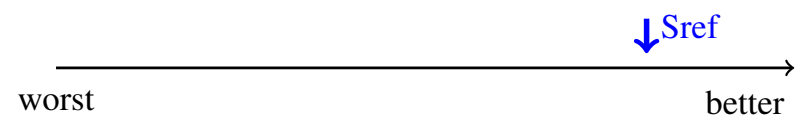

Fig. 2. The system in its reference level Sref

its reliability id spotted on fig2, on the right side of the blue arrow arbitrarily positioned.

- The real level $S_{\text {ree }}$ In developing countries, even if the system has been initially properly designed, recurrent investment delays, technically bad investments and systematic noncompliance with annual operating requirements degrade the electric system. Its reliability decreases and the system evolves from the reference level $\left(S_{\text {ref }}\right)$ to a real level $\left(S_{\text {ree }}\right)$ where the impact of constraints on the available capacity are small but significant, which means:

$$
I_{m p}(t)=\frac{\sum_{i}^{n} \delta c_{i}}{C_{A}}<1 \text { but significant }
$$




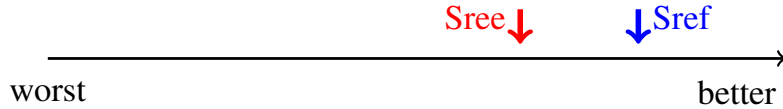

Fig. 3. The real system (Sree) versus Sref on the axis

The reliability of the system is on its real level $\left(S_{\text {ree }}\right)$ can be spotted On fig 3 , by the red arrow on the left side of the blue arrow.

b) The two status of system models: The basic requirements of simulation methods are realistic models which support a proper analysis of the system; depending on whether the requirements are met or not, the tool is appropriate or not. - The accurate model

In developing countries the electric systems are on their real levels $S_{r e e}$, let $M S_{r e l}$ be the accurate and unknown model of the system on its real level; its reliability must be spotted on fig 4 , in the vicinity of the red arrow and on left side of the blue arrow by the black arrow for instance

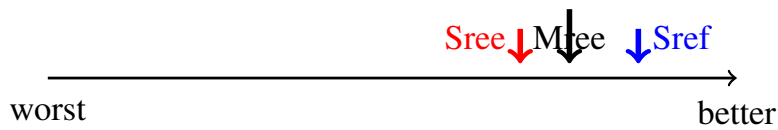

Fig. 4. The accurate system (Mree) versus Sref on the axis

- The simulated model let $M S_{\text {sim }}$ be the model used in the tool simulations; if it is realistic its reliability should be spotted on the axis, in the vicinity of the red arrow and the black one and on the left side of the blue arrow

$M S_{\text {sim }}$ is defined by the technical and economic characteristics of the system's unitary units which when $\mathrm{J}$ and $\mathrm{t}$ are respectively an exercise index and an instant $t \in J$ are translated by the equations: $\forall$ t in $J$

$$
\begin{gathered}
c_{i}(t)=\overline{c_{i}(t)}-\delta c_{i}(t)=c_{i} \\
c_{i}(t)=\overline{c_{i}(t)} \cdot\left(1-\frac{\delta c_{i}(t)}{\overline{c_{i}(t)}}\right)=c_{i} \\
\frac{\delta c_{i}(t)}{c a_{i}}<\frac{\delta c_{i}(t)}{\overline{c_{i}(t)}} \quad<<1 \quad \mid i(0<i<n)
\end{gathered}
$$

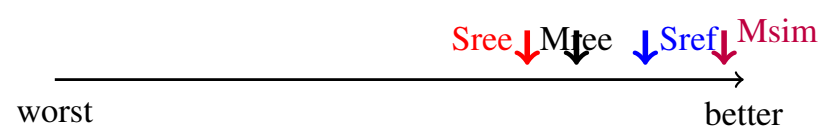

Fig. 5. The simulated model (Msim) and Mree versus Sref on the axis

c) the model is unrealistic: The Inequity 19 is valid for each unit of the system; it is more restrictive than the inequity 15 which is valid for the whole system. The reliability or the quality of the simulated model is therefore much better than that of the system in its reference level. Contrary to what is expected, one the axis fig5 the reliability of the accurate and the simulated models are spotted respectively on one side and on the other of blue arrow.

The $M S_{\text {sim }}$ is then an unrealistic model of the system in its real level, the simulations and the tools they support are both irrelevant.

\section{Conclusion}

The implementation of the management tool must should be improved in many ways; first by differentiating the interactions of the system with the different network grids and then by the definition of appropriate management indexes. However the origin of the recurrent deficiencies in the system management lay essentially on the unrealistic model of the electrical system.

The system is managed with the tool through the monitoring of the elapsed time and some of the sate quantity values of the model. The Monitored state quantities are chosen according to the management objectives, the relevant management objective indexes are results of operations between the monitored state quantity index values. A management objective index and the corresponding tool result index should then be correlated and have the same dimension.

With the unrealistic model of the electric system, it is not possible to account for the relevant management quantity indexes as results of operations between monitored state quantities values. The resulting tool indexes are not therefore correlated with the management objective indexes. The reconciliation of the dimensional analysis of the tool results and the objective management indexes in TABLE I, will give a first level of judgment on the tool's relevance.

It can be noticed on table $\mathrm{I}$ that the availability rate

TABLE I

RECONCILIATION BETWEEN MANAGEMENT QUANTITIES AND TOOL RESULTS

\begin{tabular}{|c|c|}
\hline Management goal quantities & Tool result quantities \\
\hline Reliability $\epsilon=\frac{E N D}{\text { Demand }}$ & Availability $k d=\frac{C_{\text {Adisp }}}{C_{A}}$ \\
\hline Cost $C S=\frac{\text { Monetary }}{\text { Energy }}$ & Cost $C S=\frac{\text { Monetary }}{\text { Energy }}$ \\
\hline
\end{tabular}

coefficient $(k d)$ and the corresponding reliability of the supply-demand system $\epsilon$ have the same dimension; but there are no explicit correlation between the two indexes. The management tool of the electric system is therefore irrelevant.

\section{IMPACT OF THE UNREALISTIC MODEL ON THE SYSTEM MANAGEMENT}

An unrealistic model is related to a system which is different from the actual system; the difference 
necessarily has an impact on the state variables of the system, on their variations and consequently on the system management tool.

\section{A. impact on the reference electric power value}

Before the beginning of each management exercise, systematic performance tests should be performed to determine the reference values of the state variables of each unitary element of the system.

In developing countries, considering quality of service requirements, operational constraints are so strong that systematic tests are not possible. The reference state variable values are therefore determined from the average measured values of time dependant state variables within $E_{l}$ periods of $n_{0}$ previous exercises.

The number of such $E_{l}$ periods in the duration of the planning duration $T_{p}$ is $n_{l}: n_{l}=\frac{T_{p}}{n_{0}}$, and $0<l \leq n_{l}$. The indexes of exercises in an $E_{l}$ periods are:

$\left.k_{l}=J \bmod (l-1) . n_{0}\right)$ with $0<k_{l} \leq n_{0}$.

The electric power reference variable value of unit i $C a_{i}^{E_{l}}$ is constant within $E_{l}$.

If $C_{i}^{k_{l}}(\mathrm{t})$ and $\overline{C_{i}^{k_{l}}(t)}$ are respectively the time dependant value and the yearly average of the available electric power value in the exercise $k_{l}$ :

$$
\begin{gathered}
\forall t \quad \text { in } \quad E_{l}, \quad C a_{i}^{E_{l}}>C_{i}^{k_{l}}(t) \\
C a_{i}^{E_{l}}>C a_{i}^{E_{l+1}}
\end{gathered}
$$

The variations of a single unit's electric power reference values, its time dependant values and its average values in the $n_{l} E_{l}$ periods are shown in figure 6 .

A degradation of reference values is consolidated

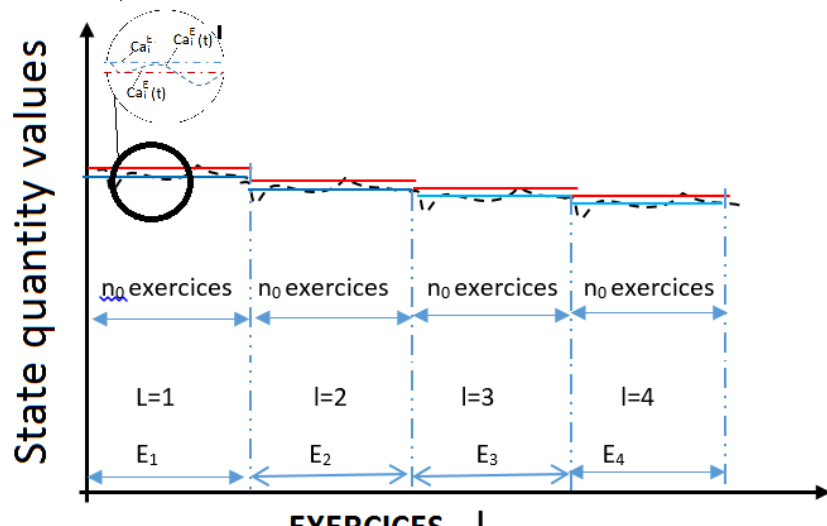

EXERCICES J

Fig. 6. Degradation of reference value consolidated by the model from one $E_{l}$ period to the next one; the degradation is not real but a consequence of the approximation,

$$
C_{i}(t)=\overline{C_{i}(t)}=C_{i}
$$

which is the ground of the model used for simulations.

Therefore when systematic tests are not possible, state quantities reference values should not be calculated as the average of the yearly measured values; on the other hand, unitary elements should be divided into $n_{t}$ groups to be tested successively each year.

\section{B. impacts on the simulations and the management tools}

Electric system have basically three independent components; a generation system component, a transmission system component and a distribution system component. Constraints are situations which result from the operational requirements or from operational incidents within one electric system component, they are characterised by an unavailable capacity $\delta C(\mathrm{t})$ which reduce the system performance. Each constraint has its beginning time $t_{d}$ and its end time $t_{f}$; in the time interval $\mathrm{T}=\left[t_{d}\right.$, $\left.t_{f}\right], \delta C(t) \neq 0$. The constraint is associated with an operational activity $\mathrm{a}(\mathrm{t})$ aimed to reduce its duration and enhance the performance of the system component.

There are several types of activities with different time characteristics

- the planned activities such as maintenance activities intended to guarantee the performance level and the system expansion activities set to match periodically the demand and the supply levels.

- the unplanned activities such as the repairing and the driving activities.

If it is assumed in the system model that the time state variables values are constant in any period of interest, the assumption means basically that the system component is invariant in that time period. It is not possible then to account for:

- the system management as results of activities, because activities cannot be defined in the time of interest period.

- the infinitesimal changes which are responsible for the evolution, the overall behaviour of the system and support the system management tool.

The model of a dynamic system, to be a realistic 
representation of the actual system, must itself be a dynamic model (i.e. time dependant). For a dynamic model, we can account for the instantaneous state quantities values, when the constraint $\delta \mathrm{C}(\mathrm{t})$ and the activity a(t) are known.

\section{Calculation of State QuAntity And PERFORMANCE VALUES}

From respectively a coarse and a fine description of the system structure, we will calculate the state quantity management and performance values of a system.

\section{A. coarse description of the system}

Let $\mathrm{t},(\mathrm{t}), \delta \mathrm{C}(\mathrm{t}), \mathrm{a}(\mathrm{t})$ and $\mathrm{u}(\mathrm{t})$, be respectively, the current time, a dynamic system, a constraint on the system, its associated activity and the resulting interaction at a given time instant $\mathrm{t}$.

1) Instantaneous quantity values

a) Instantaneous state quantity values: If $\delta \mathrm{C}(\mathrm{t})=\mathrm{O}$, we have $\mathrm{a}(\mathrm{t})=0$ and $\mathrm{u}(\mathrm{t})=(0,0) ; \mathrm{E}(\mathrm{t})=E_{0}(\mathrm{t})$ and $V_{0}(\mathrm{t})=V_{k r f}(\mathrm{t})$

$E_{0}(\mathrm{t})$ and $V_{k r f}(\mathrm{t})$ are respectively the reference state and the instantaneous state quantity reference value of $V_{k}(\mathrm{t})$.

If $\delta C(t) \neq 0$; we have $a(t) \neq 0 ; u(t)=(\delta \mathrm{C}(\mathrm{t}), \mathrm{a}(\mathrm{t}))$; The resulting interaction $\mathrm{u}(\mathrm{t})$ has an impact all the state quantities $V_{k}(\mathrm{t}): V_{k}(\mathrm{t})=V_{k r f}(\mathrm{t})+I m_{V k} \mathrm{u}(\mathrm{t})$

On applying the superposition principle:

$$
V_{k}(t)=V_{k r f}(t)+I m_{V k} \delta C(t)+I m_{V k} a(t)
$$

$I m_{V k} \delta \mathrm{C}(\mathrm{t})$ and $I m_{V k} a(\mathrm{t})$ are respectively the impact of $\delta \mathrm{C}(\mathrm{t})$ and $\mathrm{a}(\mathrm{t})$ on the state quantity $V_{k}(\mathrm{t})$. The sign of $I m_{V k} \delta \mathrm{C}(\mathrm{t})$ is negative for capacity like state quantities such as available capacity of the system and positive for input related state quantities such as the costs; while the sign of $\operatorname{Im}_{V k} a(\mathrm{t})$ is positive for cost related state quantities and zero for capacity related state quantities

According to sign of the impact $I m_{V k} u(\mathrm{t})$, there are two sets of state quantity values and performance values see fig7

- A first sets are associated with negative values of $\operatorname{Im}_{V k} \mathrm{u}(\mathrm{t})$ corresponding to $V_{k 1}(t) \leq V_{k r f}(\mathrm{t})$

- a second sets are associated with positive values of $I_{V k} \mathrm{u}(\mathrm{t})$ corresponding to $I_{k 2}(t) \geq V_{k r f}(\mathrm{t})$

An activity a(t) is associated with the constraint $\delta \mathrm{C}(\mathrm{t})$ to reduce the impact $I m_{m V k} u(\mathrm{t})$ and improve the system performance. b) Instantaneous performance values: The effectiveness of a(t) or its instantaneous performance value $P_{k}(\mathrm{t})$ is related $I m_{V k} u(\mathrm{t})$; the smaller $I m_{V k} u(\mathrm{t})$ is, the better the performance $P_{k}(\mathrm{t})$ is.

$$
\begin{gathered}
P_{k}(t)=\frac{V_{k}(t)}{V_{k} r f}(t) \\
P_{k}(t)=1+\frac{I m_{V k} u(t)}{\left.V_{k r f}(t)\right)}
\end{gathered}
$$

There are also two set of performance values associated respectively to the two sets of state quantity values. $P_{k 1}(t) \leq 1$ and $P_{k 2}(t)>1$

If $\delta C(t) \neq 0$ in a time interval $\mathrm{T}=[t 1, \mathrm{t} 2]$, with $\mathrm{t} 1$ and $\mathrm{t} 2$ respectively the debut and end instants of the constraint $\delta \mathrm{C}(\mathrm{t})$; the instantaneous state quantity values of $V_{k}(\mathrm{t})$ consolidate the management quantity values $G Q_{k r f}(\mathrm{~T})$ and $G Q_{k}(\mathrm{~T})$.

c) Management Quantities and performance values associated with an activity: The reference and the interaction dependant management quantity values are respectively:

$$
\begin{gathered}
G Q_{k r f}(T)=\int_{t 1}^{t 2} V_{k r f}(t) d t \\
G Q_{k}(T)=\int_{t 1}^{t 2}\left(V_{k r f}(t)+I m_{V k} u(t)\right) d t \\
J_{k}(T)=\int_{t 1}^{t 2} I m_{V k} u(t) d t \quad \text { is a function of } \mathrm{T}
\end{gathered}
$$

The management quantity and performance values associated with an activity are therefore:

$$
\begin{gathered}
G Q_{k}(T)=G Q_{k r f}(T)\left(1+\frac{J_{k}(T)}{G Q_{k r f}(T)}\right. \\
P_{k}(T)=\left(1+\frac{J_{k}(T)}{G Q_{k r f}(T)}\right.
\end{gathered}
$$

d) Management Quantity and performance values of in a period of interest $T$ : Most time intervals $\mathrm{T}$ are composed of a succession of nq intervals Ti such as, if $\delta C(t)=0$ in the interval period $\mathrm{Ti}$, it is positive in the following interval and vice versa.

The management quantity value in the time interval Ti is:

$$
G Q_{k}(T i)=G Q_{k r f}(T i) \cdot\left(P_{k}(T i)\right.
$$




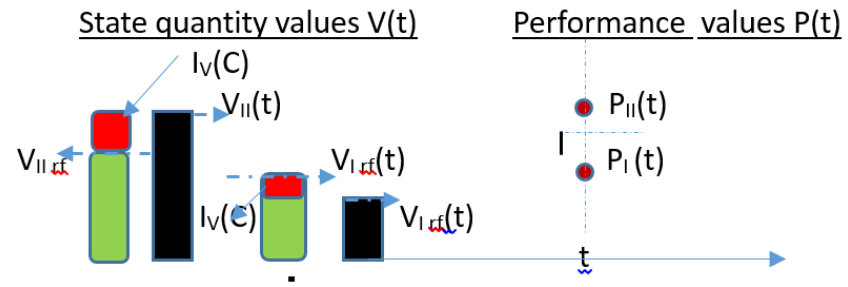

Fig. 7. The two sets of management quantity and performance values

For the whole time interval T:

$$
G Q_{k}(T)=\sum_{i=1}^{i=n q} G Q_{k r f}(T i) \cdot\left(P_{k}(T i)\right.
$$

We also have:

$$
G Q_{k}(T)=G Q_{k r f}(T) \cdot P_{k}(T)
$$

The overall performance value in $\mathrm{T}$ is therefore related to the performance of individual activities $P_{k}$ (Ti) by the relation:

$$
P_{k}(T)=\sum_{i=1}^{i=n q} \frac{G Q_{k r f}(T i)}{G Q_{k r f}(T)} \cdot\left(P_{k}(T i)\right.
$$

If $\mathrm{C} 1(\mathrm{t})=0$ within $\mathrm{T} 1(\mathrm{i}=1)$, the management quantity and performance values in odd index time intervals $\mathrm{i}=2 \mathrm{q}+1$ are respectively:

$$
G Q_{k}(T i)=G Q_{k r f}(T i)
$$

and $P_{k}(\mathrm{Ti})=1$ The management quantity and performance values in even index time intervals $i=2 q$ are respectively:

$$
G Q_{k}(T i)=G Q_{k r f}(T i) \cdot\left(P_{k}(T i)\right)
$$

and

$$
P_{k}(T i)=1+\frac{J_{k}(T i)}{G Q_{k r f}(T i)}
$$

\section{B. fine description of the system structure}

A system $\mathrm{S}$ has usually $\mathrm{n}$ unitary elements $s_{i}$; the constraint $\delta C(\mathrm{t})$ and the activities ai(t) are applied on the system through its $\mathrm{n} s_{i}$ elements fig8. A fine description of the system structure is necessary to calculate the instantaneous state quantity and performance values.

We can set the identity:

$$
(S(t), \delta C(t), a(t), u(t)) \equiv s i(t), \delta C i(t), a i(t), u i(t)
$$

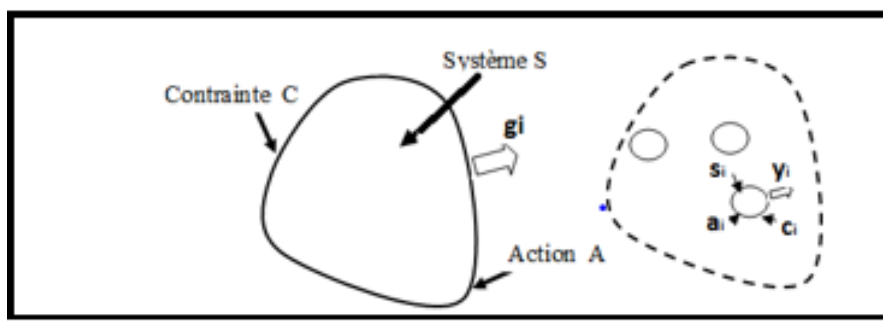

Fig. 8. Fine structure of a system

By analogy with the above coarse analysis of the system, $v i_{k r f}(\mathrm{t})$ and $v i_{k}(\mathrm{t})$ are the reference and measured values of the instantaneous state quantity $V i_{k}(\mathrm{t})$ which correspond to the constraint $\delta \mathrm{Ci}(\mathrm{t})$ and its associated activity ai $(\mathrm{t})$. The instantaneous performance value associated with the resulting interaction ui( $\mathrm{t}$ ) is:

$$
p i_{k}(t)=\left(1-\frac{\left(I m_{v k} u i(t)\right)}{v i_{k r f}(t)}\right)
$$

a) management quantity and performance values of a single activity: A single activity can be implemented on only one a single element in a time interval $\left[t i_{0}, t i_{0}+t a i\right]$, where $t i_{0}$ and tai are respectively the beginning time of ui(t) and its duration. The management quantity and the performance values associated with the state quantity $v i_{k}(\mathrm{t})$ are:

$$
\begin{aligned}
& G Q i_{k r f}(t a i)=\int_{t i_{0}}^{t i_{0}+t a i} v i_{k r f}(t) d t \\
& G Q i_{k}(t a i)=G Q i_{k r f}(t a i) . P_{k}(t a i)
\end{aligned}
$$

And :

$$
\begin{aligned}
& \left(P i_{k}(t a i)=\left(1+\frac{j i(t a i)}{G Q i_{k r f}(t a i)}\right)\right. \\
& j i(t a i)=\int_{t i_{0}}^{t i_{0}+t a i} \operatorname{Im}_{v i k} u i(t) d t
\end{aligned}
$$

$\mathrm{ji}($ tai) is the impact of ui(t) on the state quantity $v i_{k}(\mathrm{t})$ in the time interval tai

b) management quantity and performance values for several activities: Any element $s_{i}$ has, in the time interval $\mathrm{T}$, a succession of iq intervals Til such that, if $u i(t)=(0,0)$ in Til, $u i(t) \neq(0,0)$ in the following interval and vice versa.

For the whole time interval $\mathrm{T}$ the measured and the reference values of the management quantity values associated with $v i_{k}(\mathrm{t})$ are:

$$
G Q i_{k}(T)=\sum_{i l=1}^{i l=i q} G Q i_{k r f}(T i l) .\left(P Q i_{k}(T i l)\right)
$$




$$
G Q i_{k r f}(T)=\sum_{i l=1}^{i l=i q} G Q i_{k r f}(T i l)
$$

we have also

$$
G Q i_{k}(T)=G Q i_{k r f}(T)\left(P i_{k}(T)\right.
$$

The performance value of the activities on the element $s_{i}$ is:

$$
P i_{k}(T)=\sum_{i l=1}^{i l=i q} \frac{G Q i_{k r f}(T i l)}{G Q i_{k r f}(T)} \cdot\left(P i_{k}(T i l)\right)
$$

For all elements of $S(t)=\left\{s_{i}\right\}$ In the time interval $\mathrm{T}$, the time dependent management state quantity value and reference value associated with $v_{k}(\mathrm{t})$ are the sum of the corresponding values of all the elements of $\mathrm{S}(\mathrm{t})$ :

$$
\begin{gathered}
G Q_{k}(T)=\sum_{i=1}^{i=n} G Q i_{k}(T) \\
G Q_{k r f}(T)=\sum_{i=1}^{i=n} G Q i_{k r f}(T) \\
G Q_{k}(T)=\sum_{i=1}^{i=n} \sum_{i l=1}^{i l=i q} G Q i_{k r f}(T i l) .\left(P i_{k}(T i l)\right)
\end{gathered}
$$

and

$$
G Q_{k}(T)=G Q_{k r f}(T) \cdot P_{k}(T)
$$

The performance of individual activities in the time period are correlated with the overall performance $P_{k}(\mathrm{~T})$ by the relation:

$$
P_{k}(T)=\sum_{i=1}^{i=n} \sum_{i l=1}^{i l=i q} \frac{G Q i_{k r f}(T i l)}{G Q_{k r f}(T)} \cdot\left(P i_{k}(T i l)\right)
$$

\section{CONCLUSION}

Since 1982 the electric system in Developing countries use a management and are dealing with recurrent management difficulties, despite the numerous studies, diagnostics and the important investments made to improve the systems management. In this article we show that the difficulties are related to the tool implementation; first, through a description of the electrical system which is not accurate enough to account for the independent and random demands on the different voltage grids levels; and then through models of actual electrical systems which are unrealistic. The simulated models stand for invariant systems in any period of interest and lead to reference quantity value management difficulties to deal with for a reliable management of the system. They also cannot allow to set up the infinitesimal changes which lead to the overall behaviour of the system and support a good management tool implementation. Activities are the main lever of performance management, they cannot be accounted for in the framework of an invariant system; the same goes for the reference values which are essential for the notion of performance to be relevant. In this article, the simulated model is set to be dynamic or time dependant in daily, monthly, or yearly periods of interest. With the knowledge of the constraint and associated activity, associated reference or interaction free and interaction dependant instantaneous state quantity values and the instantaneous performance value are calculated. The instantaneous values consolidate the corresponding management quantity values related to the constraint and associated activity. The management quantity values for all the activities in any time of interest are calculated separately or globally to bring out an explicit correlation between the overall performance of a set of activities and the individual activities performance which is indispensable for an efficient dynamic performance management.

\section{REFERENCES}

[1] "Market survey for nuclear power in developing countries" "International Atomic International Atomic Energy Agency (IAEA)" "Vienna" "september 1973"

[2] "JENKINS, R.T. and JOY, D.S." "Wien Automatic System Planning Package (WASP) - An Electric Utility Optimal Generation Expansion Planning Computer Code" "Oak Ridge National Laboratory" " Report ORNL-4945" "1974"

[3] "MAZUMDAR, M; CHRZAN, L" , " Monte Carlo stochastic simulation of electric power generation system production costs under timedependent constraints" "Electric Power System Research" "35" "2" "November 1995" "101-108"

[4] "JENKINS, R.T., VORCE, T.C." "Use of Fourier series in the power system probabilistic simulation" "Proceeding Conference on Electric Generating System Expansion Analysis" "Columbus, Ohio state University" 1977

[5] "BONTEMPI, G;LERMAN, L" "Cours : Modélisation et simulation" "Septembre, 2015" " http://www.ulb.ac.be/di"

[6] "Expansion Planning for Electrical Generating Systems" "INTERNATIONAL ATOMIC ENERGY AGENCY" "Technical Reports Series No. 241, IAEA, Vienna" "1984"

[7] "BILLINTON, R." "Considerations in including uncertainty in LOLE calculations for practical systems" "IEEE Paper A 79 075-3 Power Engineering Society. Winter Meeting, New York" "Feb. 1979"

[8] "Model for Analysis of the Energy Demand" "INTERNATIONAL ATOMIC ENERGY AGENCY (IAEA)" "Users' Manual for Version MAED-1, -TECDOC-386, Vienna" "1986" 